\title{
A novel anti-IL4R $\alpha$ nanoparticle efficiently controls lung inflammation during asthma
}

\author{
Rabih Halwani ${ }^{1}$, Asma Sultana Shaik ${ }^{1,2}$, Elaref Ratemi ${ }^{3}$, Sibtain Afzal ${ }^{1}$, Rosan Kenana ${ }^{1,2}$, Saleh Al-Muhsen ${ }^{1}$ \\ and Achraf Al Faraj ${ }^{4}$
}

Drug resistance and the harmful side effects accompanying the prolonged corticosteroid treatment of chronic pulmonary diseases prompted the development of more specific anti-inflammatory approaches. Several strategies aiming to block IL4Ro, the receptor for a key pro-inflammatory pathway, were investigated. However, their efficiency was limited, mostly due to the systemic or subcutaneous route of administrations. In this paper, we examined the ability of an intranasal treatment with biocompatible nanoparticles targeting IL4R $\alpha$ to control lung inflammation in ovalbumin (OVA)-sensitized mice. OVA-sensitized mice were treated with anti-IL4Ro-conjugated nanoparticles. The levels of pro-inflammatory cytokines in the lungs and broncho-alveolar lavage fluid (BALF) were determined using a cytokine array assay. The effects of nanoparticle treatment on the activation of lung inflammatory cells and their ability to proliferate and produce cytokines were determined using fluorescence-activated cell sorting (FACS) analysis. Lung inflammation was also monitored using immunohistochemical staining. Treatment with the anti-IL4Ro nanoparticles significantly decreased pro-inflammatory cytokine expression and release in BALF and airway lung tissue in mice. The numbers of lung tissue lymphocytes, neutrophils and eosinophils were also decreased. Interestingly, anti-IL4Ro nanoparticles deactivated CD4 and CD8 T cells in lung tissue and inhibited their ability to produce pro-inflammatory cytokines to a significantly lower level than the treatment with free anti-IL4Ro. Moreover, they induced a sustained low level of lung inflammation for 1 week following the last instillation compared with the treatment with free anti-IL4R $\alpha$ antibodies. Together, this data suggested that the enhanced tissue penetrability and sustainability of these nanoparticles improved the strength and durability of the immunosuppressive effects of anti-IL4Ro.

Experimental \& Molecular Medicine (2016) 48, e262; doi:10.1038/emm.2016.89; published online 7 October 2016

\section{INTRODUCTION}

Corticosteroids, in use to control asthma have several limitations, including side effects and the development of drug resistance, particularly for severe cases. Alternative medications or therapeutic approaches that better control lung inflammation, tissue remodeling and asthma symptoms are urgently needed. Many molecular targets that are expected to control lung inflammation are now under intense investigation. Therapeutic strategies that aim to block unique pathways involved in asthma inflammation using specific and efficient targeted approaches could offer novel clinical treatments. One of the main methodologies tested is the inactivation of pro-inflammatory cellular pathways using blocking antibodies. However, the main limitation of this approach is the antibody administration route. The efficiency of aerosol delivery of antibodies to the lung has been limited due to several challenges. The active clearance mechanism in the lungs, which acts to remove foreign particles, markedly reduces the antibodies' residence duration. Tissue penetration is another challenge for most of the direct antibody approaches tested. Consequently, intravenous or subcutaneous administration has been the route of choice for several antibody-based clinical trials. However, the administration of antibodies via these routes may induce systemic side effects in most cases, including autoimmune diseases. Vasculitis and lupus are the most frequent anti-TNF-induced autoimmune diseases. ${ }^{1,2}$ Moreover, pulmonary diseases, such as interstitial pneumonia and sarcoid-like disorders, have also been associated with systemic monoclonal antibody (mAb) administration. ${ }^{3,4}$

\footnotetext{
${ }^{1}$ Prince Naif Center for Immunology Research and Asthma Research Chair, Department of Pediatrics, College of Medicine, King Saud University, Riyadh, Saudi Arabia; ${ }^{2}$ Prince Naif Health Research Center, King Saud University Medical City, Riyadh, Saudi Arabia; ${ }^{3}$ Jubail Industrial College, Department of Chemical and Process Engineering Technology, Jubail Industrial City, Saudi Arabia and ${ }^{4}$ Department of Radiological Sciences, College of Applied Medical Sciences, King Saud University, Riyadh, Saudi Arabia

Correspondence: Dr R Halwani, Prince Naif Center for Immunology Research and Asthma Research Chair, Department of Pediatrics, College of Medicine, King Saud University, P.O. Box 2925, Riyadh 11461, Saudi Arabia.
}

E-mail: rhalwani@ksu.edu.sa

Received 10 March 2016; revised 19 April 2016; accepted 24 April 2016 
To overcome these limitations, novel nano-sized carriers could provide a promising approach for the efficient delivery of blocking antibodies directly to asthmatic lungs. This approach could be achieved by optimizing the nanoparticles' (NPs) composition, size and structure to enhance their susceptibility and tissue penetrability. ${ }^{5}$ Of the available nanoparticles formulations, superparamagnetic iron oxide nanoparticles (SPION) have attracted extensive interest for in vivo applications due to their low intrinsic toxicity, easy surface functionalization and conjugation with targeting moieties, and ability to be readily detected using MRI. ${ }^{6}$ Recent progress in SPION design has offered new perspectives for novel magnetic nanoparticles that are capable of improving both the therapy and diagnosis in a unique multifunctional system. ${ }^{7}$ To further improve their biocompatibility for prospective clinical applications, SPION can be coated with dextran, a branched polysaccharide, which has been shown to improve tissue absorption, have a high antibody loading capacity, and exhibit sustained release. We have recently reported that surface functionalization of dextran-coated SPION with polyethylene glycol (PEG) enhances their biocompatibility and thus extends their use in safe clinical applications for the treatment of several pulmonary diseases. ${ }^{8}$

The Th2-polarized immune responses characteristic of asthmatic airway inflammation involve the dominance of IL-4 and IL-13 pro-inflammatory cytokines, key regulators of lung tissue inflammation., ${ }^{9,10}$ Both cytokines have multiple biological functions that are crucial for asthma development. ${ }^{11,12}$ IL-4 and IL-13 share the IL4R $\alpha$ subunit in their cognate receptors, and thus blocking IL4R $\alpha$ will inhibit the signaling of both cytokines. ${ }^{13}$ Several IL4R $\alpha$ blocking antibodies are being tested in phase I or II trials for their ability to efficiently control lung inflammation during asthma. ${ }^{14}$ Humanized IgG2 anti-IL4R $\alpha$ mAb administered subcutaneously was safe, well tolerated, and decreased the frequency of exacerbations; however, it did not significantly improve asthma control. ${ }^{15}$ One of the main reasons for this low efficiency could be the administration route. Besides antibodies, other receptor blockers, such as recombinant IL-4 mutein that interferes with IL4R $\alpha$ binding (Pitrakinra), was also tested in phase II clinical trials conducted on atopic asthmatics. ${ }^{16}$ Interestingly, when administered by inhalation, this mutein produced a significantly higher protective effect against allergen-challenge as well as lower FeNO levels ${ }^{16}$ compared with subcutaneous injection. This study suggested that a blocking agent may demonstrate greater efficacy when administered as an inhaled compound rather than through subcutaneous or systemic administration.

Given the potential dual suppressive effect of anti-IL4R $\alpha$ on IL-4 and IL-13 signaling and the expected improved suppressive effect of blocking agents when inhaled, the use of novel nanoparticles that would ensure efficient delivery of anti-IL4R $\alpha$ directly to the lungs could dramatically improve the efficiency of the blocking antibodies. In this paper, we have shown that PEGylated dextran SPION conjugated to anti-IL4R $\alpha$ blocking antibodies (anti-IL4R $\alpha$ NPs) efficiently suppressed lung inflammation during asthma.

\section{MATERIALS AND METHODS \\ Characterization of SPION-IL4R $\alpha$ nanoparticles}

Nanoferrite SPION with a core of $75-80 \%(w / w)$ magnetite and a shell of dextran (Micromod Partikeltechnologie GmbH, Rostock, Germany) were used. They were then cross-linked with PEG chains having a molecular weight of $2000 \mathrm{~g} \mathrm{~mol}^{-1}$ then conjugated with $13.7 \mu \mathrm{g}$ of thiolated Interleukin-4 Receptor $\alpha$ (IL4R $\alpha$ ) monoclonal antibody (BD Biosciences, CA, USA) per mg of SPION. The physicochemical properties of the developed nanoparticles, including their hydrodynamic size and zeta potential, MR relaxivities, and biocompatibility, were recently reported. ${ }^{17}$

\section{Animals and study groups}

Female BALB/c mice (18-20 g) were obtained from the main animal care facility at King Saud University. All experiments were performed in accordance with the national guidelines for the care of laboratory animals. The study was approved by the Ethical Committee of the College of Applied Medical Sciences (agreement number: CAMS07/3334). Active sensitization of mice was conducted through bi-weekly intra-peritoneal (i.p.) injections of $10 \mu \mathrm{g}$ of ovalbumin (OVA) (Bio Basic, Markham, ON, Canada) and $2.25 \mathrm{mg}$ of Alum (AlumImject; Pierce, IL, USA) in $100 \mu$ of phosphate-buffered saline. After the last sensitization (day 22), mice were challenged with $3 \%$ OVA by nebulization for three consecutive days. OVA-sensitized animals were then divided into four groups ( $n=10$ each). Mice were intrapulmonary instilled with either phosphate-buffered saline, antiIL $4 \mathrm{R} \alpha$, isotype-conjugated nanoparticles (anti-IgG1-NPs) or antiIL4R $\alpha$-NPs (Figure 2a) on days 22, 23 and 24 using a MicroSprayer aerosolizer (Penn-Century Inc., PA, USA). Anti-IgG1-NPs or antiIL4R $\alpha$-NPs were administered at a dose corresponding to $1 \mathrm{mg}$ of iron oxide per $\mathrm{kg}$ of body weight (that is, $20 \mu \mathrm{g}$ per mouse). The dose of the administered NPs has been previously optimized based on the detection threshold of MRI and its biocompatibility. ${ }^{17,18}$ The highest dose (relative to animal weight) with an excellent biocompatibility score and good MRI detection levels was chosen. Anti-IL4R $\alpha$ antibody was administered at $15 \mu \mathrm{g} \mathrm{kg}^{-1}$ (that is, $0.3 \mu \mathrm{g}$ per mouse), which corresponds to the conjugated content in the iron oxide nanoparticles. Two other non-sensitized groups of mice were treated with or without the anti-IgG1-NPs on the same time interval. Successful in vivo targeting of the nanoparticles to the inflamed lung tissue was confirmed using a combination of H\&E staining, anti-PEG antibodies, Perls' Prussian Blue reaction and immunofluorescence staining with FITC-conjugated antiIL4R $\alpha-N P s$, as previously described. ${ }^{17}$ On day 25 , BALF was collected from all groups of mice; six mice/group were killed and their lungs collected for further processing. The remaining four mice/group were killed 7 days later (day 32) and their lungs were isolated.

\section{BALF differential cell count}

The number of lymphocytes in the BALF samples collected from all treatment groups ( $n=6 /$ group) was estimated using Diff-Quick staining (Dade Behring, DE, USA). At least 100 cells/single sample were counted and the percentage of cells was determined by three independent pathologists.

\section{Cytokine analyses}

Lungs from control anti-IgG1-NPs, OVA anti-IgG1-NPs and OVA anti-IL4R $\alpha$-NPs groups of mice $(n=6)$ were cannulated through the 
trachea and lavaged three times with $1 \mathrm{ml}$ of phosphate-buffered saline containing $0.5 \mathrm{mM}$ EDTA. The levels of secreted cytokines in BALF samples were then quantified using a Cytokine multiplex assay (LMC0006 mouse 20-plex panel, Novex, Invitrogen Corporation, CA, USA) and read on Luminex-200 analyzer (Millipore, Germany). Assessments of the standards, internal controls and all samples were performed in duplicate.

\section{Gene expression studies}

Total RNA was extracted from lung tissues of the OVA anti-IgG1-NPs, and OVA-anti-IL4R $\alpha$-NPs groups of mice ( $n=6$ per treatment) (RNeasy Mini kit, Qiagen, CA, USA). Real-time PCR amplification of the cDNA targets was performed using a Power SYBR Green PCR Kit (Applied Biosystems, CA, USA). Primers specific for each cDNA (Integrated DNA Technologies, Belgium) were used to determine the expression of IL-4, IL-5, TGF- $\beta$, IFN- $\gamma$, IL-13 Eotaxin-1, muc5ac, muc2, TGF- $\beta$, MCP-4 using the 7900HT fast real-time quantitative PCR system (Applied Biosystems). GAPDH was used as an internal control. The primer sequences are shown in Supplementary Table S1. The relative expression levels of all genes of interest were normalized to GAPDH and determined by the $\Delta \Delta \mathrm{Ct}$ method using the RQ Manager Software developed by Applied Biosystems.

\section{IgE assay}

After the final challenge, serum and BALF from all treated groups were collected to measure the total IgE levels using the IgE Mouse ELISA kit (Abcam, Cambridge, UK) according to the manufacturer's instructions. The concentration of IgE in the samples was measured by reading the absorbance at $450 \mathrm{~nm}$ and then extrapolating the values from a standard curve.

\section{Isolation, stimulation and FACS analysis of lung inflammatory cells}

Peripheral blood mononuclear cells were isolated from blood samples ( $n=6$ for each group) using a Ficoll gradient (Axis Shield, Norway). To isolate lung lymphocytes, mice lungs were collected in complete RPMI-1640 medium. Lungs were minced gently in complete medium, and the filtrates containing lung lymphocytes and other inflammatory cells were collected. The viability of the freshly isolated cells was evaluated by the Trypan blue dye exclusion test. In one experiment, the samples were surface stained for $30 \mathrm{~min}$ in dark with the following anti-mouse antibodies: CD3e-PE-Cy5 $\left(0.2 \mu \mathrm{g} \mu \mathrm{l}^{-1}\right)$, CD4-APC $\left(0.1 \mu \mathrm{g} \mu \mathrm{l}^{-1}\right)$, CD8a-APC-Cy7 $\left(0.1 \mu \mathrm{g} \mu \mathrm{l}^{-1}\right)$ and the activation marker CD69-FITC $\left(0.5 \mu \mathrm{gl}^{-1}\right)$ (R\&D Systems, Minneapolis, MN, USA). In another experiment, the samples were surface stained with ITGAM FITC $\left(0.1 \mu \mathrm{g} \mathrm{ll}^{-1}\right)$ and Ly6G RPE $\left(0.1 \mu \mathrm{g} \mu \mathrm{l}^{-1}\right)$ antibodies (R\&D Systems) to determine the frequency of neutrophils (ITGAM+Ly6G+) and eosinophils (ITGAM+Ly6G-). T cells were isolated from the remaining samples using anti-CD3 magnetic beads (Miltenyi Biotec, Germany), and the cells were divided into two groups. One group was stimulated with OVA for $12 \mathrm{~h}$ (the last $3 \mathrm{~h}$ in the presence of Brefeldin A) and then intracellular cytokine staining was performed to determine ability of CD4 and CD8 cells to express cytokines. The cells were fixed in $4 \%$ paraformaldehyde, re-suspended in $0.25 \%$ saponin (S4521, Sigma-Aldrich, Germany) and stained with anti-IFN- $\gamma$ PE-Cy7 $\left(0.2 \mu \mathrm{g} \mu \mathrm{l}^{-1}\right)$, TNF- $\alpha$ PER-CP $\left(0.2 \mu \mathrm{g} \mu \mathrm{l}^{-1}\right)$, IL-4-PE-Cy7 $\left(0.2 \mu \mathrm{g} \mu \mathrm{l}^{-1}\right)$, IL-13-AF647 $\left(0.2 \mu \mathrm{g} \mu \mathrm{l}^{-1}\right)$ and IL-5 PE $\left(0.2 \mu \mathrm{g} \mu \mathrm{l}^{-1}\right)$ antibodies. The second T-cell group was stimulated with OVA for $48 \mathrm{~h}$ (the last $3 \mathrm{~h}$ in the presence of Brefeldin A), and then their proliferative capacity was determined by staining for Ki-67.
An intracellular cytokine staining assay was performed using a Ki67-FITC $\left(1 \mu \mathrm{g} \mu \mathrm{l}^{-1}\right)$ antibody (R\&D Systems). Samples were then analyzed using a BD LSR II flow cytometer equipped with DIVA (BD Biosciences) software.

\section{Histopathological analyses}

Lungs ( $n=6$ per treatment group) were obtained from the following groups of mice on days 1 and 7 following the last instillation: control anti-IgG1-NPs, OVA anti-IgG1-NPs, OVA anti-IL4R $\alpha$ and OVA antiIL4R $\alpha$-NPs. The tissues were embedded in paraffin and sets of four consecutive 5 - $\mu \mathrm{m}$-thick sections were obtained using an automatic microtome (SLEE Medical GmbH, Germany). One set of slides was stained with H\&E to assess lung inflammation following OVA sensitization and the treatments. Periodic acid-Schiff staining was used to confirm mucus production and goblet cell metaplasia in asthmatic lungs. Another set of slides were also processed for immunohistochemistry using anti-PEG antibodies (1:200 dilution; BD Pharmingen, Oxford, UK), as previously described. ${ }^{17}$

\section{Statistical analysis}

Unless otherwise specified, the data are presented as means \pm s.e. Statistical significance was evaluated using analysis of variance followed by the Bonferroni-Dunn post hoc test using SPSS v12.0 software (SPSS Inc., IL, USA). Two-way analysis of variance was performed to determine if significant differences existed in the means between different groups. $P$-values $<0.05$ were considered statistically significant.

\section{RESULTS}

\section{Efficient delivery of the anti-IL4R $\alpha$-NPs to inflamed lung tissue}

The successful synthesis and efficient targeting of nanoparticles are imperative to achieve the expected effect in the tissue environment. We have recently reported the synthesis, biocompatibility and efficient targeting of PEGylated dextran-coated anti-IL4R $\alpha$-conjugated SPION (Figure 1a) to IL4R $\alpha .{ }^{17}$ The proper binding of the anti-IL4R $\alpha-N P$ s to IL4R $\alpha$ was confirmed in vitro by measuring the competitive binding of FITC-conjugated anti-IL4R $\alpha$-NPs and non-conjugated neutralizing antibodies to IL4R $\alpha$ on murine ASM cells. ${ }^{17}$ Moreover, the efficient delivery of the instilled anti-IL4R $\alpha$ NPs into mouse lungs and its successful targeting to inflamed bronchial tissue were determined using non-invasive MRI. ${ }^{17}$ For the purpose of the current study, the localization of the instilled anti-IL4R $\alpha$-NPs within inflamed lung tissues was further confirmed using histological staining. NPs within lung tissue were detected using Perls' PB staining (stains Iron $(\mathrm{Fe})$ ) (Figure 1b(ii)), anti-PEG antibodies (Figure $1 \mathrm{~b}($ iii)) or immunofluorescence to detect the FITC-labeled anti-IL4R $\alpha$ NPs (Figure $1 \mathrm{~b}(\mathrm{iv})$ ). As expected, the anti-IL4R $\alpha$-NPs localized within areas of lung tissue enriched in inflammatory cells, as confirmed by $\mathrm{H} \& \mathrm{E}$ staining (Figure $1 \mathrm{~b}(\mathrm{i})$ ). This data confirmed the efficient targeting of anti-IL4R $\alpha$-NPs to inflamed lung tissues of the treated mice. 
a

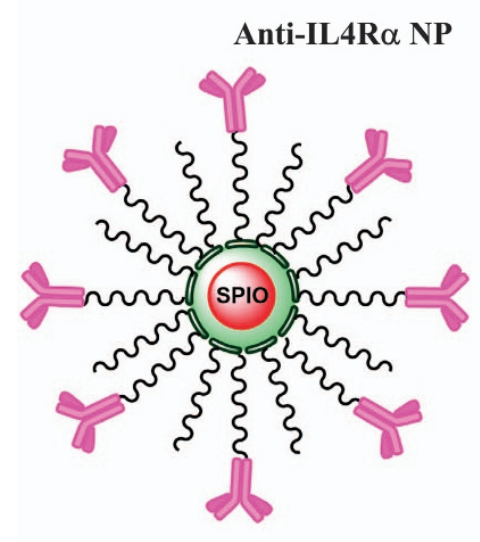

b
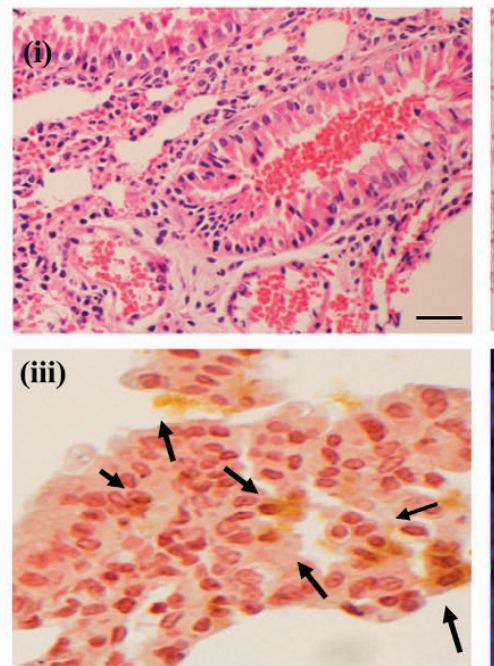

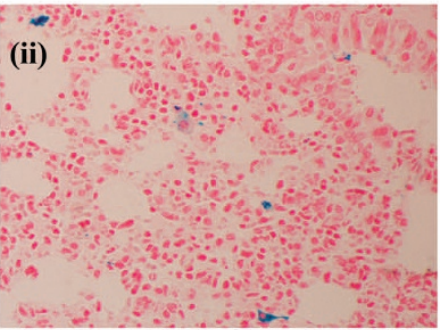

(iv)

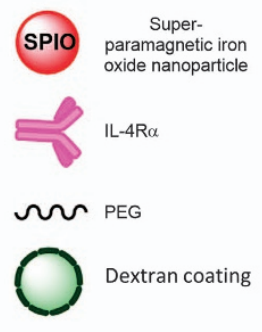

Figure 1 Proper administration of anti-IL4R $\alpha$-NPs to inflamed lung tissue. (a) A diagram representing the synthetized anti-IL4R $\alpha$ nanoparticles. (b) Histological assessments confirming the targeting of anti-IL4R $\alpha$ NPs to the inflamed tissue in asthmatic lungs. (i) H\&E staining showing tissue inflammation, (ii) Prussian blue staining (marker for iron oxide) showing distribution of SPION within the inflamed tissue and (iii) immunohistochemical staining for PEG using anti-PEG antibodies also confirmed the presence of SPION. The black arrows indicate the localization of the PEGylated nanoparticles. (iv) Immunofluorescence staining showing the presence of the FITC-anti-IL4R $\alpha$ NPs within the tissue. Immunofluorescence images were captured at an exposure of $300 \mathrm{~ms}$. Scale bar, $100 \mu \mathrm{m}$.

\section{Anti-IL4R $\alpha$-NPs effectively downregulate inflammatory cytokine expression}

To evaluate the efficiency of anti-IL4R $\alpha$-NPs in suppressing lung tissue inflammation in vivo, OVA-sensitized mice were instilled with or without anti-IL4R $\alpha$-NPs, anti-IL4R $\alpha$ antibodies or control anti-IgG1-NP, as described in Figure 2a. The control non-sensitized groups were also treated with or without anti-IgG1-NPs (Figure 2a). BALF and lung tissue of the antiIL4R $\alpha$-NP-treated mice was collected $24 \mathrm{~h}$ after the last instillation and analyzed to determine the inflammatory cytokine levels. Treating the OVA-sensitized and nonsensitized mice with anti-IgG1-NPs did not affect cytokine gene or protein levels compared with the non-treated group (data not shown). However, treating OVA-sensitized mice with the anti-IL4R $\alpha$-NPs resulted in a significant decrease in the BALF levels of IL-6, IL-17, GM-CSF, IL-5, TNF- $\alpha$, MCP-1, IL-12p40/70, IL-4, IL-13, IFN- $\gamma$ and IL-2 cytokines compared with the OVA-sensitized anti-IgG1-NP-treated mice (Figure 2b, Table 1). To substantiate the anti-inflammatory effect of the anti-IL4R $\alpha$-NPs, the expression of selected pro-inflammatory genes within the lung tissue, including IL-4, IL-5, TGF- $\beta$, IFN- $\gamma$, IL-13 Eotaxin- 1 , muc5ac, muc2, TGF- $\beta$, MCP-4, was determined. A significant decrease in the relative expression levels of IFN- $\gamma$ (2.14 vs $0.77, P=0.01)$, IL-4 (1.12 vs $0.86, P=0.05)$, IL-5 (2.56 vs $1.16, P \leqslant 0.001)$, IL-13 (1.98 vs $1.01, P \leqslant 0.001)$, CCL-11 (4.47 vs $1.11, P \leqslant 0.001)$, Muc5ac (1.58 vs $1.01, P=0.05)$ and Muc2 (2.79 vs $1.11, P \leqslant 0.001)$ was noted following instillation with the anti-IL4R $\alpha$-NPs compared with the OVA-sensitized, anti-IgG1-NP-treated mice (Figure 2c). There was no effect on the MCPT-4 (1.17 vs 1.11, $P=\mathrm{NS}$ ) and TGF- $\beta$ (1.29 vs $1.03, P=\mathrm{NS}$ ) levels. These results indicated that treatment with the anti-IL4R $\alpha$-NPs efficiently suppressed the expression of key pro-inflammatory genes within the inflamed lung tissue.

Anti-IL4R $\alpha$-NPs reduce the number of BALF inflammatory cells and IgE levels

BALF profiles of inflammatory cells and mediators, including the IgE levels, represent a good indicator of lung tissue inflammation during asthma. ${ }^{19,20}$ BALF was collected from all groups of mice (six mice/group) on day 25 and analyzed to determine the IgE levels and numbers of inflammatory cells. Treating the OVA-sensitized mice with anti-IL4R $\alpha$-NPs significantly decreased the frequency of lymphocytes $(21.0 \%$ vs $9.0 \%, P<0.01)$ compared with the OVA-sensitized, anti-IgG1-NP-treated mice (Figure 3a). Moreover, a significant decrease in the $\operatorname{BALF}\left(1.31\right.$ vs $0.47 \mathrm{ng} \mathrm{ml}^{-1}, P<0.01$ ) and serum (5.74 vs $\left.4.34 \mathrm{ng} \mathrm{ml}^{-1}, P<0.05\right)$ IgE levels was also observed in the asthmatic mice treated with the anti-IL4R $\alpha$ NPs (Figure 3b). These BALF findings revealed that the anti-IL4R $\alpha$-NPs could reduce lung tissue inflammation during asthma.

Anti-IL4R $\alpha$-NPs efficiently control lung tissue inflammatory cell activation, proliferation and function during asthma The observed ability of anti-IL4R $\alpha$-NPs to downregulate the expression of inflammatory mediators indicated that these particles may deactivate and suppress the function of inflammatory cells in lung tissue. To investigate this possibility, inflammatory cells were isolated from the lungs of mice (six mice/group) collected on day 25. The activation and proliferation of CD4 and CD8 cells and their ability to secrete cytokines were examined. The frequencies of eosinophils and neutrophils were also determined. A significant decrease in 

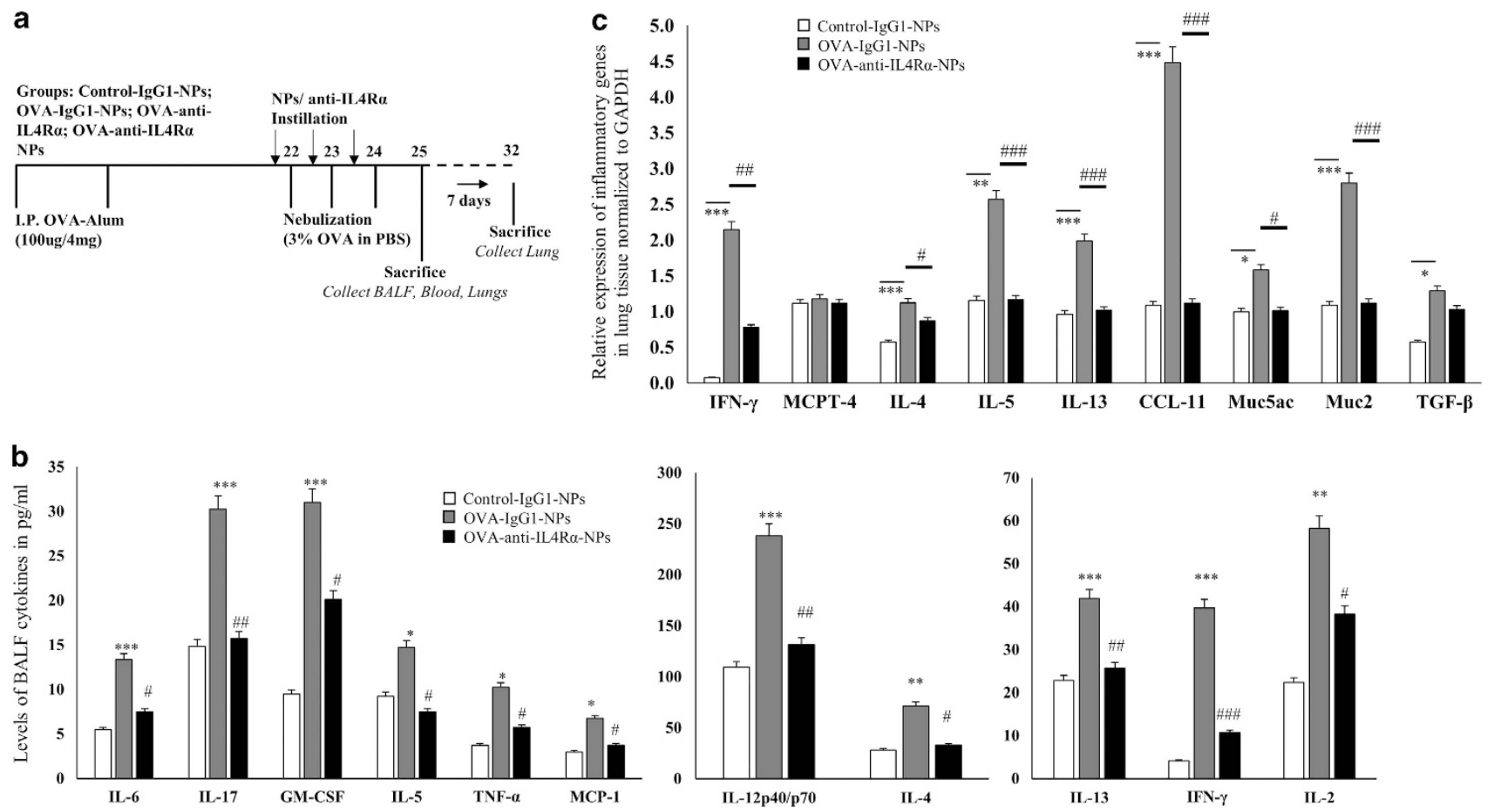

Figure 2 Effect of anti-IL4R $\alpha$-NPs on the expression of lung pro-inflammatory cytokines in the treated mice. (a) Study design for the in vivo treatment of the OVA-sensitized mice with the nanoparticles. (b) BALF levels of pro-inflammatory cytokines in mice treated with or without the anti-IL4R $\alpha$-NPs. The cytokine levels were estimated using a mouse cytokine multiplex array. (c) Expression of inflammatory cytokine genes within the lung tissues of mice treated with anti-lgG1-NPs or anti-IL4R $\alpha$-NPs. Relative gene expression normalized to GAPDH is presented. $\left({ }^{*}\right)$ for control vs OVA, ${ }^{*} P<0.05,{ }^{* *} P<0.01,{ }^{* * *} P<0.001$; $\left({ }^{\#}\right)$ for OVA vs anti-IL4R $\alpha$-NPs, ${ }^{\#} P<0.05,{ }^{\# \#} P<0.01$, \#\#\# $P<0.001$.

Table 1 BALF levels of pro-inflammatory cytokines are significantly decreased following treatment with the anti-IL4Ra-NPs

\begin{tabular}{|c|c|c|c|c|}
\hline Cytokines & Control-IgG1-NPs (pg m/-1) & OVA-lgG1-NPs (pg m/-1) & OVA-IL4Ra-NPs $\left(\mathrm{pg} \mathrm{ml^{-1 }}\right)$ & P-value (OVA-lgG1-NPs vs OVA-IL4Ra-NPS) \\
\hline IL-17 & $14.87 \pm 1.8$ & $30.25 \pm 4.3$ & $15.75 \pm 2.3$ & $P=0.01$ \\
\hline IL-5 & $9.25 \pm 1.3$ & $14.75 \pm 1.5$ & $7.5 \pm 0.8$ & $P=0.01$ \\
\hline TNF- $\alpha$ & $3.75 \pm 0.6$ & $10.25 \pm 1.4$ & $5.75 \pm 1.2$ & $P<0.05$ \\
\hline MCP-1 & $3 \pm 0.9$ & $6.75 \pm 1.7$ & $3.75 \pm 0.4$ & $P=0.003$ \\
\hline $\mathrm{IFN}-\gamma$ & $4.12 \pm 1.3$ & $39.75 \pm 3.8$ & $10.75 \pm 1.7$ & $P<0.001$ \\
\hline IL-2 & $22.37 \pm 3.2$ & $58.25 \pm 4.1$ & $38.25 \pm 3.6$ & $P=0.01$ \\
\hline IL-12p40/70 & $109.5 \pm 6.9$ & $238 \pm 7.8$ & $131.75 \pm 5.8$ & $P<0.001$ \\
\hline IL-4 & $28.12 \pm 2.4$ & $72.62 \pm 3.2$ & $32.87 \pm 2.6$ & $P=0.05$ \\
\hline
\end{tabular}

Data is presented as means \pm s.e.

CD69 expression, a marker for T-cell activation, was observed in lung $\mathrm{T}$ cells of mice treated with free anti-IL4R $\alpha$ antibodies $(43.3 \%$ vs $58.6 \%, P=0.05)$ compared with OVA-anti-IgG1NP-treated controls. However, a more dramatic decrease was observed following treatment with the anti-IL4R $\alpha$-NPs $(32.1 \%$ vs $58.6 \%, P<0.001)$. In fact, CD69 expression was significantly reduced on the T cells from the anti-IL4R $\alpha-\mathrm{NP}$-treated mice compared with those from the free anti-IL4R $\alpha$-treated group $(32.1 \%$ vs $43.3 \%, P=0.04)$ (Figure $4 a)$. Similarly, the proliferative ability of CD4 and CD8 cells isolated from mice treated with anti-IL4R $\alpha$ NPs was significantly reduced compared with either those isolated from the OVA-stimulated anti-IgG1-NP-treated mice (CD4 5.2\% vs $14.6 \% ; P<0.001$, CD8 $3.1 \%$ vs $13.8 \% ; P<0.001$ and $\mathrm{CD}^{+} \mathrm{CD}^{+} 7.3 \%$ vs $19.3 \% ; P<0.001)$ or mice treated with free anti-IL-4R $\alpha$ antibodies (CD4 $5.2 \%$ vs $11.8 \%$, CD8 $3.1 \%$ vs $11.3 \%$; $P<0.01$, and $\mathrm{CD}^{+} \quad \mathrm{CD}^{+} \quad 7.3 \%$ vs $18.2 \%, \quad P<0.05$; Figure $4 \mathrm{~b}$ ). These data indicated that anti-IL4R $\alpha$-NPs are much more efficient in controlling T-cell proliferation than free anti-IL4R $\alpha$.

Anti-IL4R $\alpha$-NPs are expected to deactivate T cells and thereby hinder their capacity to produce inflammatory 

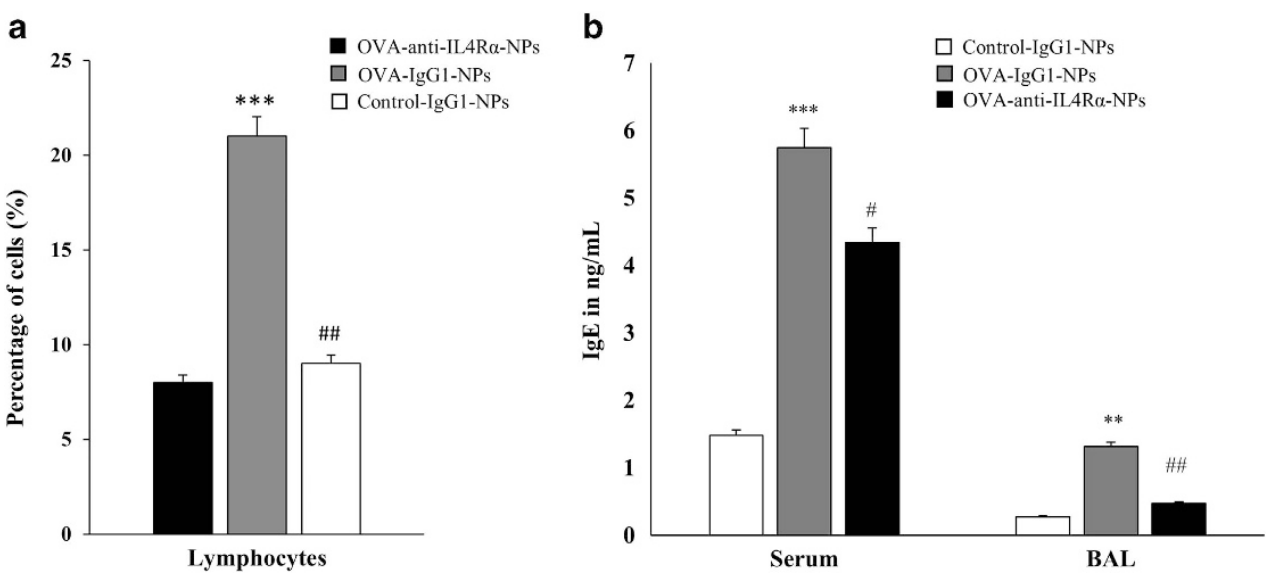

Figure 3 Effects of the anti-IL4R $\alpha-N P$ s on the BALF inflammatory cells and IgE levels in the treated mice. (a) A significant decrease was observed in the frequency of BALF lymphocytes following treatment with anti-IL4R $\alpha$-NPs, as determined by fluorescence-activated cell sorting (FACS) analysis. (b) Serum and BALF IgE levels in the control and anti-IL4R $\alpha$-NP-treated mice. $\left({ }^{*}\right)$ for control vs OVA, ${ }^{* *} P<0.01$, ${ }^{* * *} P<0.001 ;\left({ }^{\#}\right)$ for OVA vs anti-IL4R $\alpha-N P s,{ }^{\#} P<0.05,{ }^{\# \#} P<0.01, \# \# P<0.001$.

cytokines. To investigate this possibility, the ability of CD4 and CD8 $\mathrm{T}$ cells isolated from the lung tissues of all mice groups to express IL-4, IL-5, IFN- $\gamma$ and TNF- $\alpha$ upon OVA stimulation was determined. Treating OVA-stimulated mice with antiIL4R $\alpha$-NPs significantly suppressed the ability of CD4 cells to express inflammatory cytokines upon OVA stimulation (CD4: IL-4: $17.1 \%$ vs $57.7 \%, P<0.001$; IL-5: $0.9 \%$ vs $5.1 \%, P<0.01$; TNF- $\alpha$ : $5.2 \%$ vs $6.9 \%, P=0.05$; IFN- $\gamma: 14.1 \%$ vs $22.5 \%$, $P=0.02$ ). Treating mice with free anti-IL4R $\alpha$ also inhibited the ability of CD4 cells to produce cytokines in response to OVA stimulation, but to a lesser extent compared with the anti-IL4R $\alpha$ NPs (except for IFN- $\gamma$ and TNF- $\alpha$ production) (Figure 4c). A similar suppression of cytokine expression was also observed for CD8 cells treated with the anti-IL4R $\alpha$-NPs (CD8: IL-4: $13.8 \%$ vs $28 \%, P<0.001$; IL-5: $6.9 \%$ vs $9.2 \%$, $P=0.05$; TNF- $\alpha: 4.7 \%$ vs $6.8 \%, P=0.04$; IFN- $\gamma: 13.8 \%$ vs $30.4 \%, P<0.01)$. This suppression was somewhat higher than that resulting from treating CD8 cells with free anti-IL4R $\alpha$ (IL-4: $13.8 \%$ vs $19.3 \%, P=0.05$; IFN- $\gamma^{+}: 13.2 \%$ vs $18.9 \%$, $P=0.05$ ), with the exception of TNF- $\alpha$ (Figure $4 \mathrm{~d}$ ). Furthermore, the frequencies of neutrophils and eosinophils were reduced following treatment with the anti-IL4R $\alpha$-NPs (neutrophils: $41.8 \%$ vs $84.3 \%, P=0.01$; eosinophils: $31.3 \%$ vs $62.1 \%, P<0.01)$ compared with OVA-sensitized, anti-IgG1NP-treated mice (Figure 4e). Interestingly, this treatment was more efficient than free anti-IL4R $\alpha$ in reducing the number of eosinophils in the lung tissue. These data indicated that anti-IL4R $\alpha$-NPs suppressed lung inflammatory cell activation, proliferation and pro-inflammatory cytokine production to more significant level than free anti-IL4R $\alpha$.

\section{Anti-IL4R $\alpha$-NPs controlled lung tissue inflammation and mucus production}

To evaluate the ability of anti-IL4R $\alpha$-NPs to maintain sustained control of lung tissue inflammation during OVA-induced asthma, histological assessments were performed for all groups of mice on days 2 and 7. One day following the last instillation,
anti-IL4R $\alpha$-NP- and free anti-IL4R $\alpha$-treated mice had clear bronchiolar lumens and nearly normal mucosal and sub-mucosal layers, in contrast to the increased infiltration of inflammatory cells and mucus production observed for the OVA-sensitized anti-IgG1-NP-treated mice (Figure 5a). The proper distribution of NPs within the lung tissue was confirmed using an anti-PEG antibody. On day 7 post instillation (day 32), lung inflammation resumed for mice treated with the free anti-IL4R $\alpha$ antibodies. An obstruction of the bronchioles lumens by mucoid exudates, goblet cell metaplasia and increased numbers of infiltrating inflammatory cells near sub-mucosal layers were observed. Interestingly, however, the anti-IL4R $\alpha$-NP-treated lungs continued to sustain a low level of inflammation as shown in the histological profile of the airway tissues, with decreased numbers of inflammatory cells, mucus production and goblet cell metaplasia (Figure 5b). These data suggested that the anti-IL4R $\alpha$-NPs were more efficient than free anti-IL4R $\alpha$ antibodies in suppressing inflammation and in maintaining this therapeutic effect for longer durations.

\section{DISCUSSION}

The airway geometry, humidity, pulmonary epithelium and specific defense mechanisms in the lungs, such as the mucociliary escalator, macrophage and enzymatic activities, represent challenges to drug delivery. ${ }^{21}$ Although several formulation strategies have been tested, including the use of protease inhibitors, surfactants and permeation-enhancing polymers, ${ }^{22,23}$ there is still a need to improve delivery efficiency, targeting specificity and decrease the side effects. Nanoparticlebased drug delivery systems targeting the lungs offer many advantages over other applications. This is mostly due to their decreased mucociliary clearance and long retention time in the lungs, their ability to release drugs at a controlled rate, and their biodegradability. ${ }^{24}$ The advantage of magnetic NPs over other NPs (for example, micelles, liposomes, and so on) is that their magnetic properties allow their quantitative biodistribution to be monitored in the target tissue using 
a
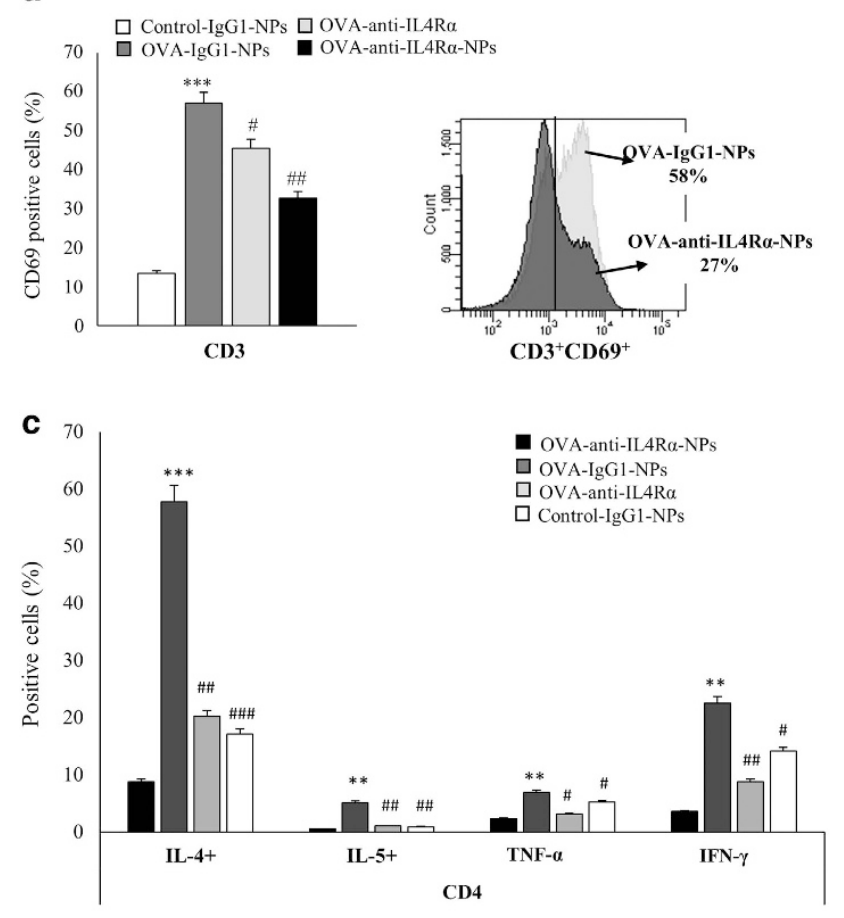

b

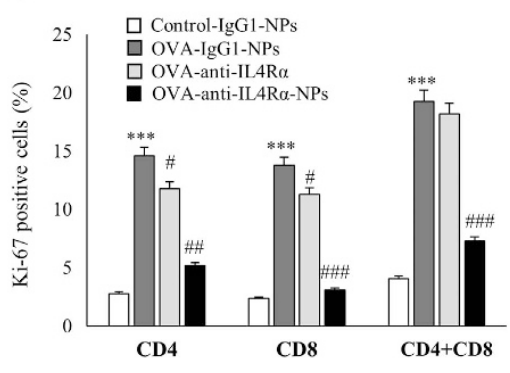

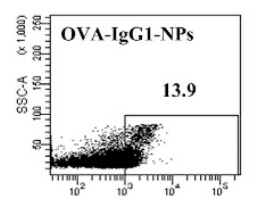

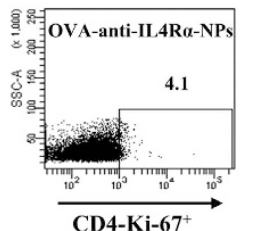

CD4-Ki-67+

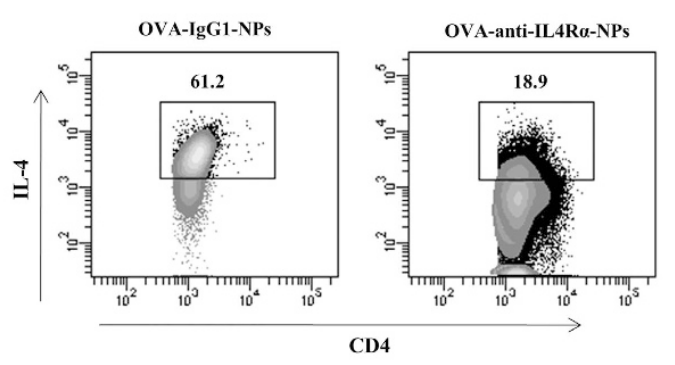

e
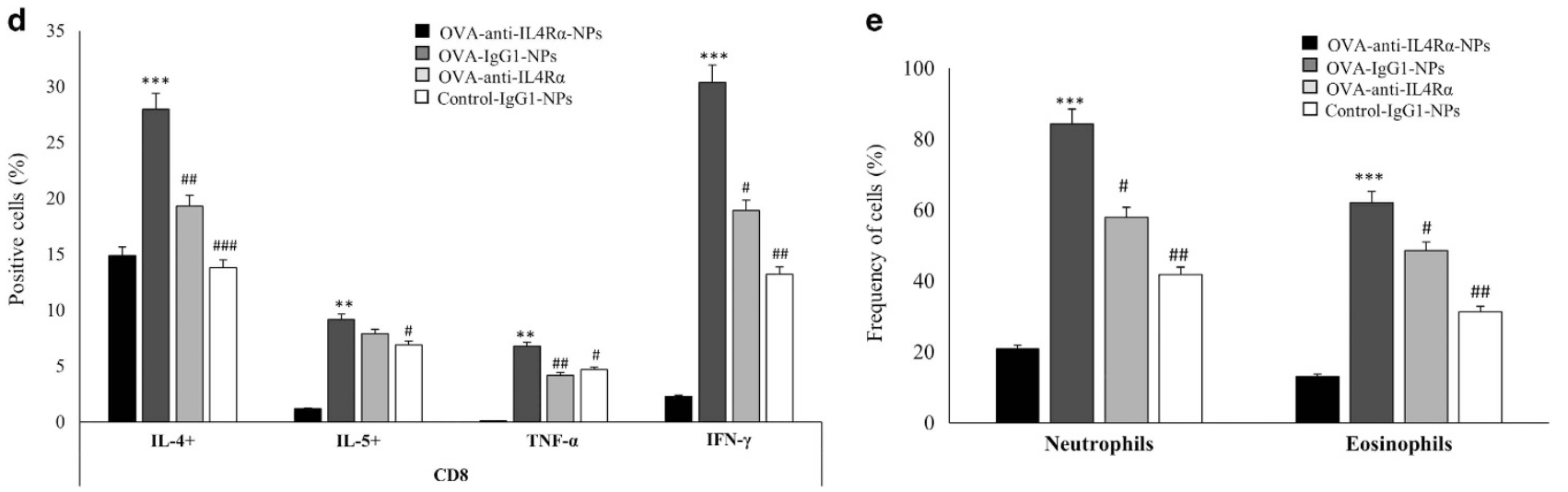

Figure 4 Effects of the anti-IL4R $\alpha$-NPs on lung inflammatory cell activation and function in the treated mice. (a) CD69 expression level on CD3 cells in the lung tissue before and after the anti-IL4R $\alpha$-NP treatment. Representative FACS data showing a decrease in CD69 expression following treatment with the anti-IL4R $\alpha-N P S$. (b) Effect of the anti-IL4R $\alpha$-NP treatment on the proliferative ability of CD4 and CD8 T cells in response to OVA stimulation. CD4 and CD8 cells isolated from the lungs of mice treated with or without the anti-IL4Ro-NPs were stimulated with OVA for 2 days. The Ki-67 levels were determined using FACS analysis. Representative FACS data showing the decrease in Ki-67 expression in CD4 cells following treatment with the anti-IL4R $\alpha$-NPs. (c, d) CD4 and CD8 cells isolated from the lungs of mice treated with or without the anti-IL4R $\alpha$-NPs cells were stimulated with OVA and the frequency of cells producing IL-4, IL-5, TNF- $\alpha$ and IFN- $\gamma$ was determined using FACS analysis. Representative FACS data showing the decrease in CD4 cells expressing IL-4 following treatment with anti-IL4R $\alpha$-NPs. (e) Frequencies of neutrophils and eosinophils isolated from the lungs of mice treated with or without anti-IL4R $\alpha$-NPs. $\left.{ }^{*}\right)$ for control vs OVA, ${ }^{* *} P<0.01,{ }^{* *} P<0.001 ;\left({ }^{\#}\right)$ for OVA vs anti-IL4R $\alpha-N P s,{ }^{\#} P<0.05,{ }^{\# \#} P<0.01,{ }^{\# \# \# P<0.001 . ~}$

noninvasive MRI. ${ }^{25}$ We and others have successfully used MRI to visualize the inflamed lung tissue by quantifying edema, a characteristic feature of lung inflammation, in mice $^{26}$ by detecting the hyper-intense pixels. ${ }^{17}$ Currently, a number of SPION are in early clinical trials or experimental study stages, ${ }^{6}$ and several formulations have been approved for clinical use in medical imaging and therapeutic applications.

We have recently reported the biocompatibility of PEGylated dextran-coated anti-IL4R $\alpha$-conjugated SIPON and their efficient binding and proper targeting to IL4R $\alpha .{ }^{17} \mathrm{PEG}$ exhibits a $\mathrm{pH}$-sensitive behavior, which insures that it will dissociate in an acidic tissue environment, such as in inflamed tissue ( $\mathrm{pH}=6.4-6.8),{ }^{27}$ leading to the release of the associated blocking antibodies. ${ }^{17}$ This property of the herein described NPs is expected to ensure the proper release and delivery of anti-IL4R $\alpha$ blocking antibodies in the inflamed lung tissues. In addition, PEG functionalization has been used to reduce the phagocytic capture of NPs by the immune system, ${ }^{28}$ and hence 

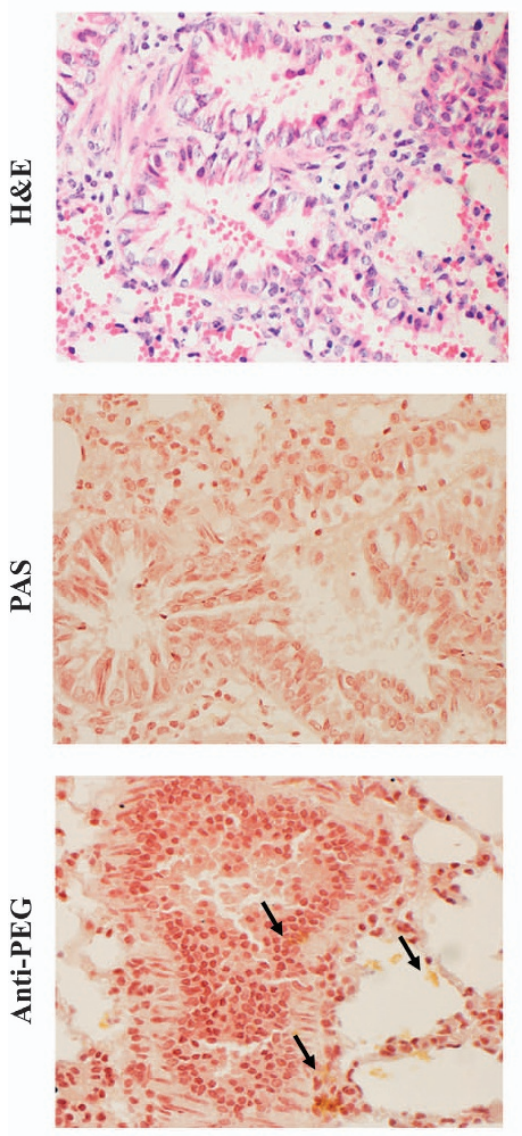

b
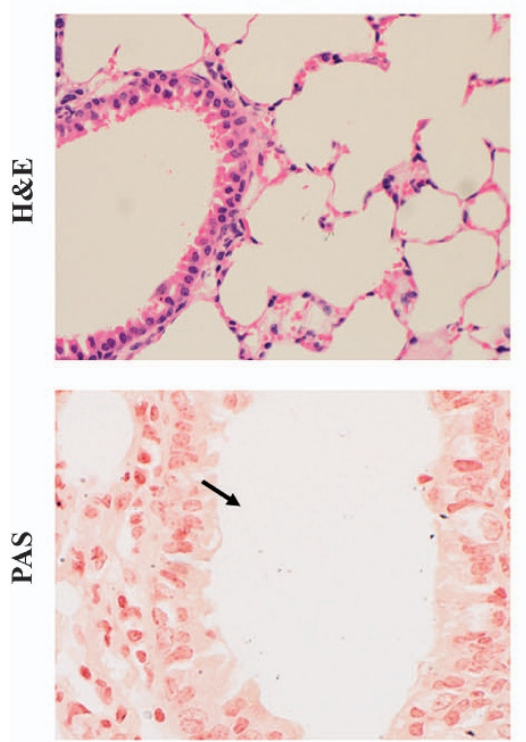

OVA-anti-IL4R $\alpha$
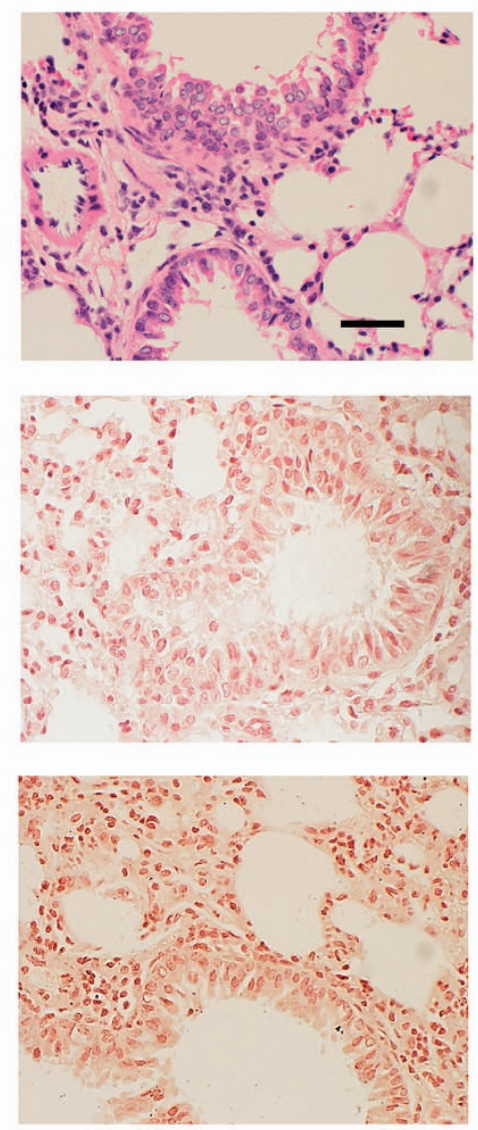

OVA-anti-IL4R $\alpha$
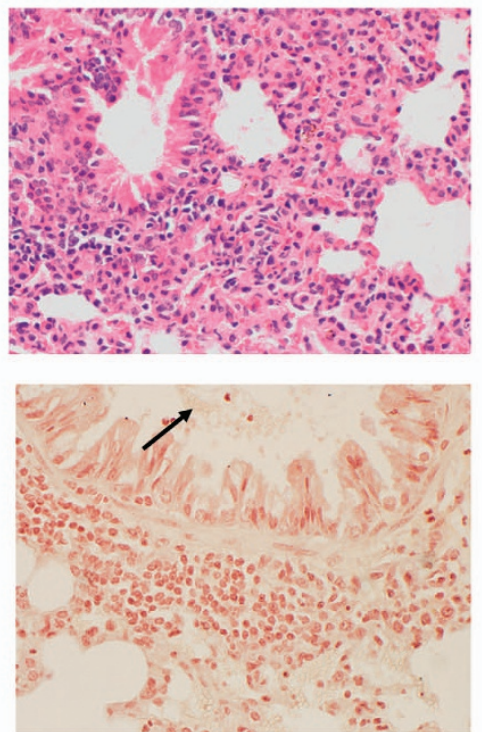

OVA-anti-IL4R $\alpha$ NPs
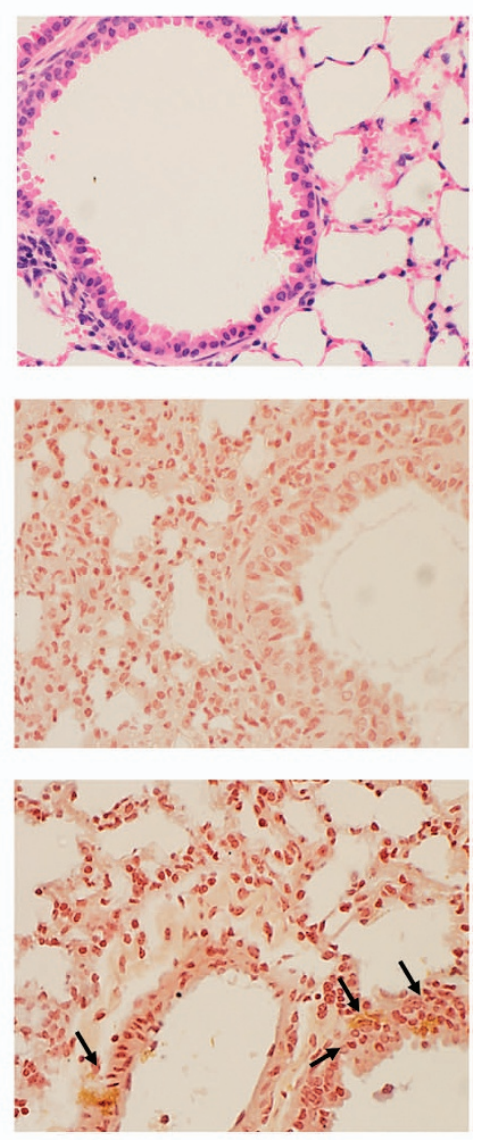

OVA-anti-IL4R $\alpha$ NPs
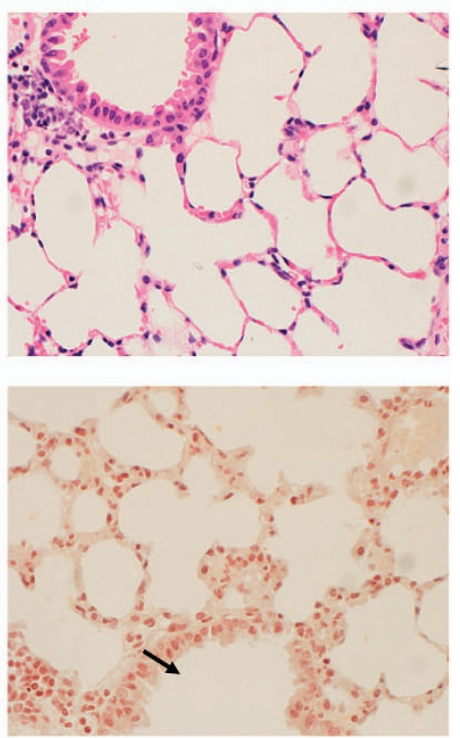

Figure 5 Treatment with anti-IL4R $\alpha$-NPs induces sustained control of lung inflammation. (a) Lung sections of OVA-sensitized mice treated with anti-IgG1-NPs, free anti-IL4R $\alpha$ and anti-IL4R $\alpha$-NPs. The sections were isolated $24 \mathrm{~h}$ following the last instillation. The upper panels are stained with H\&E, the middle panels show Periodic acid Schiff staining (for mucus hypersecretion) and the lower panels show immunohistochemical staining with an anti-PEG antibody to show the tissue distribution of the NPs. (b) Histological assessment of lung sections obtained 7 days following the last instillation with either free anti-IL4R $\alpha$ or anti-IL4R $\alpha$-NPs. The upper panels are stained with H\&E, and the lower panels show periodic acid-Schiff staining. In contrast to treatment with free anti-IL4R $\alpha$, where Iung inflammation and obstruction of the bronchiole lumen through mucus accumulation resumed 7 days following the last instillation, lungs treated with anti-IL4R $\alpha$-NPs maintained a low level of inflammation and mucus clearance. Scale bar, $100 \mu \mathrm{m}$. 
is expected to enhance the sustainability of the anti-IL4R $\alpha$-NPs and their subsequent accumulation in the targeted tissue.

Biological compounds targeting IL4R $\alpha$ may provide a new therapeutic modality, especially for patients with uncontrolled, severe asthma. ${ }^{15,29}$ Strategies enabling the direct administration of these blocking agents/antibodies to the inflamed lung tissue are expected to both improve the treatment efficacy and decrease the systemic side effects. In this study, we have shown that anti-IL4R $\alpha$-NPs efficiently control lung inflammation. Intrapulmonary treatment of the OVA-sensitized mice with anti-IL4R $\alpha$-NPs markedly reduced the pro-inflammatory cytokine levels in the lung tissue. However, treatment with anti-IgG1-NPs had neither immunogenic nor suppressive effects. In addition to the reduction in the cytokine levels, a significant decrease in the frequency and proliferation of the lung inflammatory cells was observed. Although treatment with free anti-IL4R $\alpha$ induced a significant suppression in the cells' functionality, the anti-IL4R $\alpha$-conjugated NPs were generally more efficient in controlling cytokine release. Interestingly, in contrast to free anti-IL4R $\alpha$, these nanoparticles maintained a sustained control of lung tissue inflammation and mucus production 7 days following treatment. PEGylated dextrancoated NPs are known for their ability to cross biological barriers by transiently opening tight junctions between epithelial cells, escaping macrophage phagocytosis, and ensuring excellent biodistribution and sustainability. ${ }^{30}$ As expected, these nanoparticles exhibited improved efficiency, sustainability and hence an overall increase in the anti-inflammatory effect of the IL4R $\alpha$ antibodies. Further investigations are required to determine the effectiveness of IL4R $\alpha$-NPs in maintaining control of lung inflammation for prolonged periods and will be the subject of a future communication.

Signaling through IL4R $\alpha$ by either IL- 4 or IL-13 activates signal transducer and activator of transcription factor-6 (STAT-6). ${ }^{31}$ Most of the pathological and physiological manifestations during allergic disorders, including Th2 cell differentiation, IgE synthesis, mucus cell metaplasia and airway hyper responsiveness (AHR), depend on STAT- 6 . The fact that STAT-6 activation is critical for the differentiation of naive $\mathrm{T}$ cells into Th2 effector cells, as well as IL-4 production, ${ }^{32}$ explains the suppression of Th2 cytokines upon treatment with anti-IL4R $\alpha$ NPs observed in this study. Moreover, downregulation of IgE levels in the serum and BALF of the anti-IL4R $\alpha$-NP-treated mice was expected, as IL-4 signaling is critical for the production of IgE. ${ }^{33}$ In addition, IL4R $\alpha$ and IL-4 cytokines were shown to promote CD4 and CD8 T-cell proliferation, respectively. ${ }^{34,35}$ This result explains the decrease in CD4 and CD8 proliferation following treatment with anti-IL4R $\alpha$-NPs.

A more recent approach used subcutaneous administration of Dupilumab, a humanized anti-IL4R $\alpha$ monoclonal antibody, to treat severe asthmatic patients with elevated eosinophil levels. ${ }^{36}$ The treatment was associated with fewer asthma exacerbations, improved lung function and reduced levels of Th2-associated inflammatory markers, even with the withdrawal of LABAs and inhaled glucocorticoids. ${ }^{36}$ Signaling through IL4R $\alpha$ was also shown to promote eosinophil maturation $^{37}$ and to enhance IL-5 cytokine production. ${ }^{38}$ These findings could explain the significant decrease in the secretion of IL-5 cytokine by CD4 and CD8 cells following treatment with anti-IL4R $\alpha$-NPs observed in this study. In fact, this observation could be the reason behind the corresponding decrease in the frequency of lung eosinophils. This specific ability of the NPs to control eosinophils and IL-5, the cytokine regulating their production and survival, is particularly important for the treatment of asthmatic patients with high eosinophil counts. However, further experiments are needed to confirm this observation.

Blocking IL4R $\alpha$ signaling using various approaches, including anti-IL4R $\alpha$ antibodies, has been shown to decrease mediators of inflammation and inflammatory cell counts in BALF and lung tissue, as well as serum IgE levels. ${ }^{15,16}$ This finding agrees with our observations in this study showing the effects of anti-IL4R $\alpha$-NPs. AHR, as measured by methacholine challenge, was also shown to be reduced. ${ }^{15,16}$ Moreover, phase I/II clinical trials using the nebulized, soluble, extracellular portion of IL4R $\alpha$ to treat mild-to-moderate persistent asthma patients showed improved asthma symptom scores, improved FEV1 and decreased AHR..$^{39}$ One limitation of our study is that we were not able to measure the effect of anti-IL4R $\alpha$-NP treatment on AHR because a respiratory function measurement system was not available in our facility. However, the efficient suppression of inflammatory cell activation and inflammatory mediator release following treatment with the NPs suggest that this approach may efficiently control AHR.

In conclusion, the optimized anti-IL4R $\alpha$-NPs developed here were more efficient in controlling lung inflammation than free anti-IL4R $\alpha$. The use of biocompatible nanoparticles for drug delivery offers a promising approach not only for inhibiting inflammatory pathways but also for the targeted treatment of the pathological structural changes characteristic of chronic inflammation. This property could be of special value for the treatment of airway smooth muscle hyperplasia or epithelial cell destruction during late, severe stages of asthma. Therefore, this approach could pave the way for various anti-inflammatory and drug delivery applications in asthma, as well as other inflammatory diseases, including COPD, rhinitis and pulmonary fibrosis. A safe and targeted delivery system could readily improve the performance of classical medicines and offers a strategic tool to expand the current drug markets. Moreover, the rapid development of the nanoparticle field, particularly in imaging applications, suggests that this approach could also potentially be used to monitor lung tissue inflammation and tissue remodeling during chronic inflammatory diseases.

\section{CONFLICT OF INTEREST}

The authors declare no conflict of interest.

\section{ACKNOWLEDGEMENTS}

This project was funded by the National Plan for Science, Technology and Innovation (MAARIFAH), King Abdulaziz City for Science and Technology, Kingdom of Saudi Arabia, Award Number (12-MED2536). 
1 Ramos-Casals M, Brito-Zeron P, Munoz S, Soria N, Galiana D, Bertolaccini $\mathrm{L}$ et al. Autoimmune diseases induced by TNF-targeted therapies: analysis of 233 cases. Medicine 2007; 86: 242-251.

2 Ramos-Casals M, Brito-Zeron P, Cuadrado MJ, Khamashta MA. Vasculitis induced by tumor necrosis factor-targeted therapies. Curr Rheumatol Rep 2008; 10: 442-448.

3 Ramos-Casals M, Roberto Perez A, Diaz-Lagares C, Cuadrado MJ, Khamashta MA, BIOGEAS Study Group. Autoimmune diseases induced by biological agents: a double-edged sword? Autoimmun Rev 2010; 9: 188-193.

4 Soubrier M, Jeannin G, Kemeny JL, Tournadre A, Caillot N, Caillaud D et al. Organizing pneumonia after rituximab therapy: two cases. Joint Bone Spine 2008; 75: 362-365.

5 Grill AE, Johnston NW, Sadhukha T, Panyam J. A review of select recent patents on novel nanocarriers. Recent Pat Drug Deliv Formul 2009; 3: 137-142.

6 Jin R, Lin B, Li D, Ai H. Superparamagnetic iron oxide nanoparticles for MR imaging and therapy: design considerations and clinical applications. Curr Opin Pharmacol 2014; 18: 18-27.

7 Laurent S, Saei AA, Behzadi S, Panahifar A, Mahmoudi M. Superparamagnetic iron oxide nanoparticles for delivery of therapeutic agents: opportunities and challenges. Expert Opin Drug Deliv 2014; 11 1449-1470.

8 Al Faraj A, Shaik AP, Shaik AS. Effect of surface coating on the biocompatibility and in vivo MRI detection of iron oxide nanoparticles after intrapulmonary administration. Nanotoxicology 2015; 9: 825-834.

9 Wills-Karp M, Luyimbazi J, Xu X, Schofield B, Neben TY, Karp CL et al. Interleukin-13: central mediator of allergic asthma. Science 1998; 282: 2258-2261.

10 Robinson DS, Hamid Q, Ying S, Tsicopoulos A, Barkans J, Bentley AM et al. Predominant TH2-like bronchoalveolar T-lymphocyte population in atopic asthma. N Engl J Med 1992; 326: 298-304.

11 Kopf M, Le Gros G, Bachmann M, Lamers MC, Bluethmann H, Kohler G. Disruption of the murine IL-4 gene blocks Th2 cytokine responses. Nature 1993; 362: 245-248.

12 Punnonen J, Aversa G, Cocks BG, McKenzie AN, Menon S, Zurawski G et al. Interleukin 13 induces interleukin 4-independent IgG4 and IgE synthesis and CD23 expression by human B cells. Proc Natl Acad Sci USA 1993; 90: 3730-3734.

13 Borish L. IL-4 and IL-13 dual antagonism: a promising approach to the dilemma of generating effective asthma biotherapeutics. Am J Respir Crit Care Med 2010; 181: 769-770.

14 Oh CK, Geba GP, Molfino N. Investigational therapeutics targeting the IL-4/ IL-13/STAT-6 pathway for the treatment of asthma. Eur Respir Rev 2010; 19: 46-54.

15 Corren J, Busse W, Meltzer EO, Mansfield L, Bensch G, Fahrenholz J et al. A randomized, controlled, phase 2 study of AMG 317, an IL-4Ralpha antagonist, in patients with asthma. Am J Respir Crit Care Med 2010; 181: 788-796.

16 Wenzel S, Wilbraham D, Fuller R, Getz EB, Longphre M. Effect of an interleukin-4 variant on late phase asthmatic response to allergen challenge in asthmatic patients: results of two phase 2a studies. Lancet 2007; 370: $1422-1431$.

17 Al Faraj A, Shaik AS, Afzal S, Al-Muhsen S, Halwani R. Specific targeting and noninvasive magnetic resonance imaging of an asthma biomarker in the lung using polyethylene glycol functionalized magnetic nanocarriers. Contrast Media Mol Imaging 2015; 11: 172-183.

18 Al Faraj A, Shaik AS, Al Sayed B, Halwani R, Al Jammaz I. Specific targeting and noninvasive imaging of breast cancer stem cells using single-walled carbon nanotubes as novel multimodality nanoprobes. Nanomedicine (Lond) 2016; 11: 31-46.

19 Barnes PJ. The cytokine network in asthma and chronic obstructive pulmonary disease. J Clin Invest 2008; 118: 3546-3556.

20 Yssel H, Abbal C, Pene J, Bousquet J. The role of IgE in asthma. Clin Exp Allergy 1998; 28(Suppl 5): 104-109.

21 Hastings RH, Folkesson HG, Matthay MA. Mechanisms of alveolar protein clearance in the intact lung. Am J Physiol Lung Cell Mol Physiol 2004; 286: L679-L689.
22 Jalalipour M, Najafabadi AR, Gilani K, Esmaily H, Tajerzadeh H. Effect of dimethyl-beta-cyclodextrin concentrations on the pulmonary delivery of recombinant human growth hormone dry powder in rats. J Pharm Sci 2008; 97: 5176-5185.

23 Hussain A, Arnold JJ, Khan MA, Ahsan F. Absorption enhancers in pulmonary protein delivery. J Control Release 2004; 94: 15-24.

24 Tsapis N, Bennett D, Jackson B, Weitz DA, Edwards DA. Trojan particles: large porous carriers of nanoparticles for drug delivery. Proc Natl Acad Sci USA 2002; 99: 12001-12005.

25 Jain TK, Reddy MK, Morales MA, Leslie-Pelecky DL, Labhasetwar V. Biodistribution, clearance, and biocompatibility of iron oxide magnetic nanoparticles in rats. Mol Pharm 2008; 5: 316-327.

26 Al Faraj A, Shaik AS, Afzal S, Al Sayed B, Halwani R. MR imaging and targeting of a specific alveolar macrophage subpopulation in LPS-induced COPD animal model using antibody-conjugated magnetic nanoparticles. Int J Nanomedicine 2014; 9: 1491-1503.

27 Tsuchiya H, Mizogami M, Ueno T, Takakura K. Interaction of local anaesthetics with lipid membranes under inflammatory acidic conditions. Inflammopharmacology 2007; 15: 164-170.

28 Larsen EK, Nielsen T, Wittenborn T, Birkedal H, Vorup-Jensen T, Jakobsen $\mathrm{MH}$ et al. Size-dependent accumulation of PEGylated silane-coated magnetic iron oxide nanoparticles in murine tumors. ACS Nano 2009; 3: 1947-1951.

29 Brightling CE, Saha S, Hollins F. Interleukin-13: prospects for new treatments. Clin Exp Allergy 2010; 40: 42-49.

30 Azarmi S, Roa WH, Lobenberg R. Targeted delivery of nanoparticles for the treatment of lung diseases. Adv Drug Deliv Rev 2008; 60: $863-875$.

31 Hershey GK. IL-13 receptors and signaling pathways: an evolving web. J Allergy Clin Immunol 2003; 111: 677-690.

32 Kaplan MH, Schindler U, Smiley ST, Grusby MJ. Stat6 is required for mediating responses to IL-4 and for development of Th2 cells. Immunity 1996; 4: 313-319.

33 Deo SS, Mistry KJ, Kakade AM, Niphadkar PV. Role played by Th2 type cytokines in IgE mediated allergy and asthma. Lung India 2010; 27: 66-71.

34 You D, Marr N, Saravia J, Shrestha B, Lee GI, Turvey SE et al. IL-4Ralpha on $\mathrm{CD}^{+} \mathrm{T}$ cells plays a pathogenic role in respiratory syncytial virus reinfection in mice infected initially as neonates. J Leukoc Biol 2013; 93: 933-942.

35 Morris SC, Heidorn SM, Herbert DR, Perkins C, Hildeman DA, Khodoun MV et al. Endogenously produced IL-4 nonredundantly stimulates CD8+ T cell proliferation. J Immunol 2009; 182: 1429-1438.

36 Wenzel S, Ford L, Pearlman D, Spector S, Sher L, Skobieranda F et al. Dupilumab in persistent asthma with elevated eosinophil levels. $N$ Engl J Med 2013; 368: 2455-2466.

37 Fulkerson PC, Schollaert KL, Bouffi C, Rothenberg ME. IL-5 triggers a cooperative cytokine network that promotes eosinophil precursor maturation. J Immunol 2014; 193: 4043-4052.

38 Knott ML, Matthaei KI, Foster PS, Dent LA. The roles of eotaxin and the STAT6 signalling pathway in eosinophil recruitment and host resistance to the nematodes Nippostrongylus brasiliensis and Heligmosomoides bakeri. Mol Immunol 2009; 46: 2714-2722.

39 Borish LC, Nelson HS, Corren J, Bensch G, Busse WW, Whitmore JB et al. Efficacy of soluble IL-4 receptor for the treatment of adults with asthma. J Allergy Clin Immunol 2001; 107: 963-970.

(c) (1) () $\Theta$ This work is licensed under a Creative Commons Attribution-NonCommercial-NoDerivs 4.0 International License. The images or other third party material in this article are included in the article's Creative Commons license, unless indicated otherwise in the credit line; if the material is not included under the Creative Commons license, users will need to obtain permission from the license holder to reproduce the material. To view a copy of this license, visit http://creativecommons.org/licenses/by-nc-nd/4.0/

Supplementary Information accompanies the paper on Experimental \& Molecular Medicine website (http://www.nature.com/emm) 